
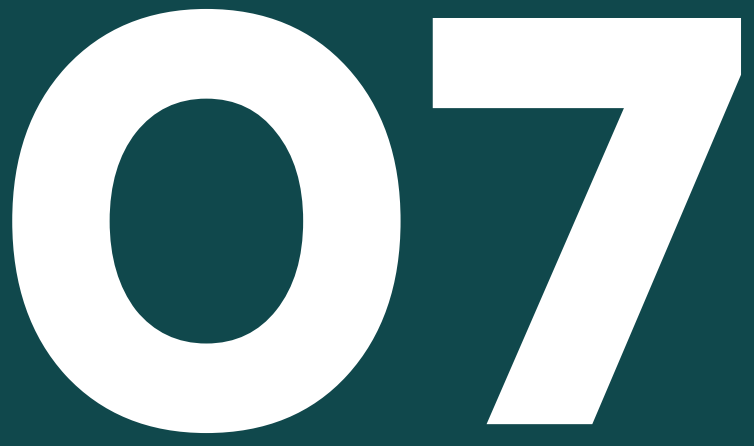

\title{
Análisis de los programas de responsabilidad social en la imagen corporativa del sector cooperativista de la provincia de Tungurahua
}

Analysis of social responsibility programs in the corporate image of the cooperative sector of the province of Tungurahua

FECHA DE RECEPCIÓN: 21/05/2021

FECHA DE APROBACIÓN: 17/08/2021

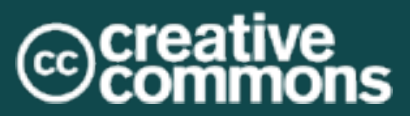




\section{Resumen}

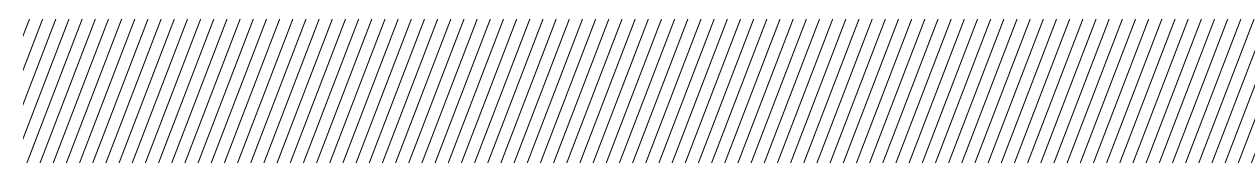

\section{Elias David Caisa Yucailla ${ }^{1}$ Jefferson Stalin Tapia Vasquez ${ }^{2}$}

Las organizaciones pertenecientes a la economía popular y solidaria son responsables por el cumplimiento de las expectativas que tienen los socios sobre las implicaciones de sus inversiones en los ámbitos medioambientales y la comunidad. La presente investigación tuvo por objetivo determinar el impacto de la responsabilidad social en la construcción de una imagen corporativa positiva en las cooperativas de ahorro y crédito del segmento 3 y 4 de la provincia de Tungurahua. Se aplicó una encuesta a los gerentes de 37 instituciones financieras para evaluación de su imagen corporativa, socios externos, socios internos e inversionistas. La confiabilidad para la aplicación del instrumento fue de 96\%. Los hallazgos revelaron que, las instituciones han descuidado al grupo de interés externo e inversionistas. Sin embargo, se evidenció que las cooperativas de ahorro y crédito mantienen su responsabilidad hacia sus empleados (socios internos); es decir, el bienestar de sus socios internos ha sido su prioridad, pues, ha ocasionado el fortalecimiento interno de la organización.

Palabras clave: Responsabilidad social, imagen corporativa, sector cooperativista, stakeholders.

1 Universidad Técnica de Ambato · eliasdcaisa@uta.edu.ec · https://orcid.org/0000-0003-41684800 - Ambato, Ecuador

2 Universidad Técnica de Ambato · Jeff.tapia2014@gmail.com • https://orcid.org/0000-0003-

0319-355X - Ambato, Ecuador 


\section{Abstract}

rganizations belonging to the popular and solidarity economy are responsible for meeting the expectations that members have regarding the implications of their investments in the environmental and community spheres. The objective of this research was to determine the impact of social responsibility in the construction of a positive corporate image in the savings and credit cooperatives of segment 3 and 4 of the province of Tungurahua. A survey was applied to the managers of 37 financial institutions to evaluate their corporate image, external partners, internal partners, and investors. The reliability for the application of the instrument was $96 \%$. The findings revealed that the institutions have neglected the external interest group and investors. However, it was evidenced that savings and credit cooperatives maintain their responsibility towards their employees (internal partners), that is, the well-being of their internal partners has been their priority, since it has caused the internal strengthening of the organization.

Keywords: Social responsibility, corporate image, cooperative sector, stakeholders.

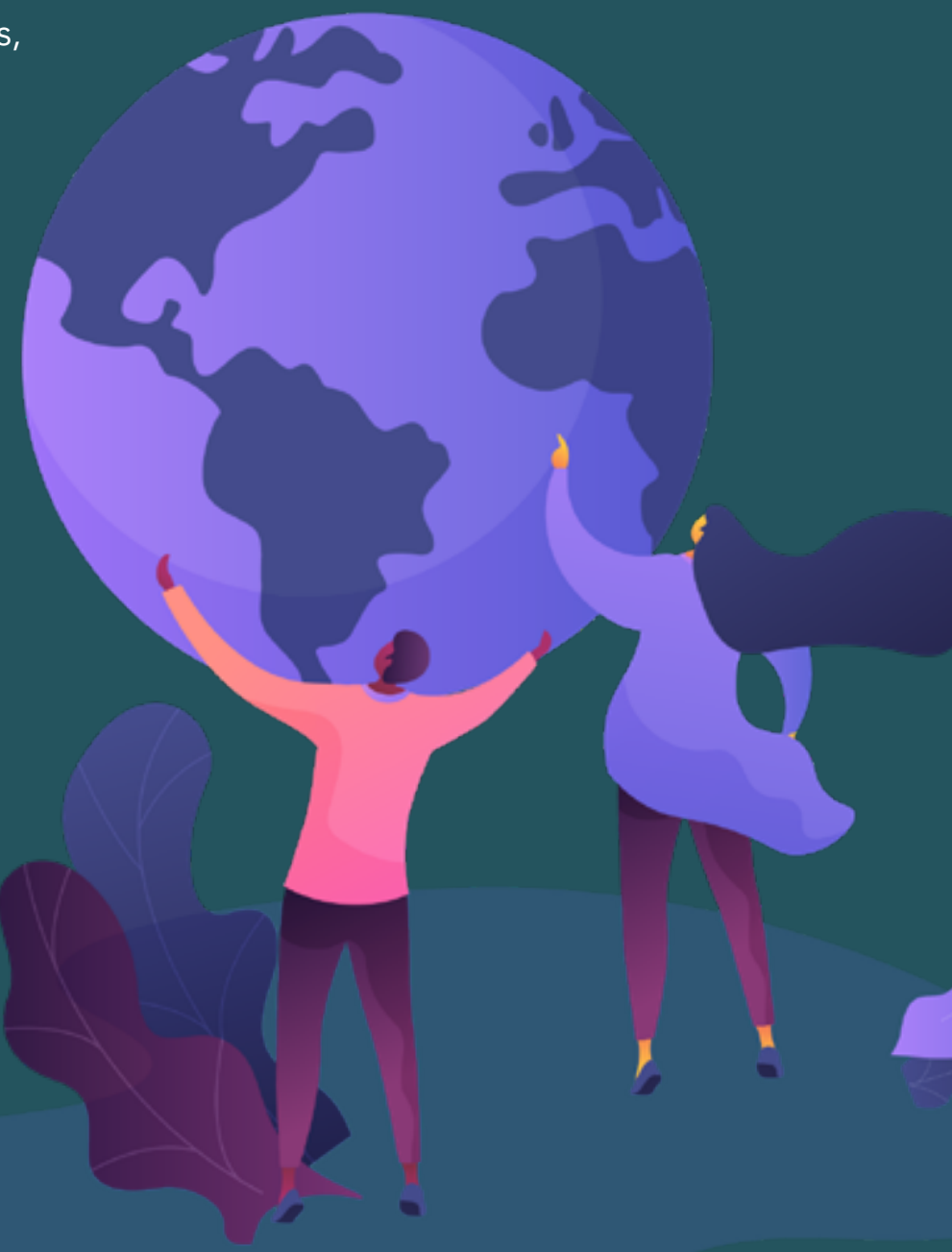




\section{Introducción}

finales del siglo XX, lamentablemente, varias organizaciones se vieron envueltas en escándalos empresariales debido a acciones irresponsables y poco éticas. Por ejemplo, en el caso de Enron y Nestlé se evidenció la inexistencia de mecanismos reguladores del actuar administrativo de los altos ejecutivos encargados de las decisiones corporativas (Martín \& Aroca, 2016). De ahí, las empresas empiezan a tomar conciencia sobre las implicaciones que tienen sus acciones, no solo dentro del ámbito económico, sino también, social y medioambiental (Coba et al., 2017).

A partir de los años setenta, la Responsabilidad Social Corporativa (RSC) toma auge. Diversos colectivos empiezan a demandar mayor información sobre el comportamiento social empresarial (Server \& Villalonga, 2015). Con el surgimiento del internet y el acceso a la información, los clientes son más conscientes y exigen mayor compromiso por parte de las organizaciones, mejor calidad de los productos, servicios que cuiden la salud de los consumidores y demandan operaciones que no dañen el medio ambiente (Schwalb, 2011).

En América Latina, los consumidores muestran un favoritismo hacia la intervención gubernamental de obligar a las empresas a manejar sus actividades con responsabilidad social (Schwalb, 2011). Por lo mismo, es necesario incluir buenas prácticas de gobierno y adoptar medidas de RSC, las cuales, deben alinearse a las estrategias empresariales de cada organización (Martín \& Aroca, 2016)corporate social responsibility (CSR.

A pesar de no existir una definición general de RSC, existen varios aspectos comunes en torno a este tema, como: el comportamiento ético de la organización, las condiciones laborales y el impacto en el medio ambiente. De igual manera, hoy en día una imagen corporativa positiva, es una muestra de una gestión empresarial eficaz. Un componente esencial para alcanzar dichos propósitos es la RSC; por tal motivo, es necesario utilizar estrategias que fortalezcan la imagen y reputación de una empresa (Garcia \& Llorente, 2009). 
La implementación de programas de responsabilidad social ha supuesto un gran avance para muchas empresas, puesto que, ser socialmente responsable produce una gran ventaja, ya que genera un impacto directo en la productividad empresarial. Además, con una política de RSC no solo da cumplimiento a las obligaciones jurídicas, también se aplican normas más estrictas en el ámbito social para mejorar las condiciones laborales y la relación entre la dirección y los trabajadores (Correa, 2007).

Varias investigaciones aportan sobre el impacto que concibe la imagen corporativa, a través de la responsabilidad social corporativa. De acuerdo con Garcia \& Llorente (2009) en su estudio titulado, La responsabilidad social corporativa: una estrategia para conseguir Imagen y reputación, hacen mención de la importancia de construir una imagen positiva con base al desarrollo de la RSC. En este sentido, las políticas de RSC y una adecuada comunicación pueden consolidar y mantener una imagen corporativa positiva. De esta manera, las actividades socialmente responsables repercuten directamente en las actividades económicas propias de cada empresa.

Del mismo modo, Awan, Hayat, \& Faiz (2018) en su estudio de Antecedentes y consecuencias de la imagen corporativa: Bancos convencionales e Islámicos, revelan que, la inversión en actividades de RSC contribuyen directamente a la imagen corporativa. Con ello, se desarrolla una mejor actitud hacia la marca y la adquisición de nuevos servicios. El estudio revela que una mejor comunicación empresarial crea un vínculo de familiaridad con la marca y mejorar la imagen corporativa de los bancos, lo cual, beneficia a los resultados empresariales de dichas instituciones.

En el Ecuador, la responsabilidad social es un tema de interés tanto para instituciones públicas como privadas. En su mayoría, las prácticas de RSC son llevadas a cabo, principalmente, por grandes empresas o sociedades multinacionales; sin embargo, es igual de importante implementar políticas de RSC en todo tipo de empresas y sectores económicos. La ejecución de prácticas sociales en las micro, pequeñas y medianas empresas, es fundamental porque son las que más contribuyen a la economía y a la creación de puestos de trabajo (Vélez \& Cano, 2016).

Las empresas realizan o colaboran con proyectos de responsabilidad social corporativa, tal es el caso de Banco del Pichincha, Corpora- 
ción Favorita, Holcim, entre otras, que cuentan con fundaciones benéficas dedicadas a la labor social. En cambio, en el caso de las cooperativas de ahorro y crédito no se cuenta con una base de datos completa sobre el tipo de proyectos y los valores económicos asignados a cada uno.

En el año 2019, varias instituciones de la economía popular y solidaria llevaron a cabo talleres de capacitación y mantuvieron convenios con varias instituciones benéficas para brindar apoyo a sectores vulnerables, por ejemplo la Cooperativa de ahorro y crédito "Ambato" aportó $55.537,79$ dólares a proyectos de responsabilidad social enfocados al área medioambiental, educativo y deportivo (COAC Ambato, 2020); no obstante, no se cuenta con mayor información sobre las demás instituciones y sus aportes en el balance social.

La presente investigación tiene por objetivo demostrar el impacto que genera la responsabilidad social en la construcción de la imagen corporativa de las Cooperativas de Ahorro y Crédito de los segmentos 3 y 4 de la provincia de Tungurahua. Mediante la aplicación de una encuesta a los gerentes corporativos, se pretende corroborar las siguientes hipótesis (ver figura 1).

Figura 1. Cuadro hipotético

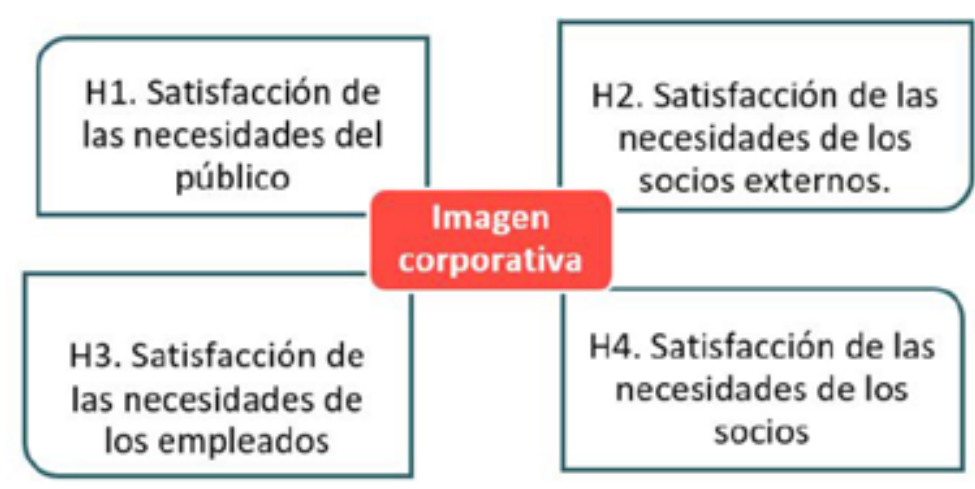

Fuente: Elaboración propia. 
H1. La satisfacción de las necesidades del público se relaciona positivamente con la imagen corporativa.

H2. La satisfacción de las necesidades de los socios externos se relaciona positivamente con la imagen corporativa.

H3. La satisfacción de las necesidades de los empleados (socios internos) se relaciona positivamente con la imagen corporativa.

H4. La satisfacción de las necesidades de los inversionistas se relaciona positivamente con la imagen corporativa.

Las hipótesis establecidas fueron comprobadas y se estableció, si existe o no, una relación positiva entre la implementación de programas de responsabilidad social y la actitud hacia la imagen corporativa.

\section{Responsabilidad Social Corporativa}

El desarrollo sostenible se ha convertido en un factor importante dentro de las empresas. Con frecuencia, diversos colectivos promueven iniciativas ligadas a la RSC, como una forma de cumplir con los requisitos de sustentabilidad que mejorará su reputación ante la sociedad (Zhivkova, 2019). La RSC surge con la aparición de pequeñas instituciones filantrópicas de países como Reino Unido y Francia. A inicios del siglo XX, no existe dicho término como tal, no obstante, las diversas instituciones son quienes brindan soluciones a los problemas sociales que pueden plantearse.

En la primera mitad del siglo XX, ya se inicia la realización de acciones filantrópicas puntuales; es así como, en 1919, se instauró la Organización Internacional del Trabajo (OIT) con el propósito de mejorar las condiciones de trabajo de los empleados. Posteriormente, en la segunda 
mitad del siglo XX, la comunidad empieza a tomar conciencia sobre la capacidad del sector privado, para dar solución a problemas sociales que se ocasionan en el entorno (Cardona, 2017).

De acuerdo con el Manual de balance social se entiende como RSC, la respuesta que la institución debe dar a las expectativas en los sectores con los cuales tiene relación como, el desarrollo integral de los trabajadores y el aporte a la comunidad (ANDI, 2001). Así también, del Rio (citado por Ochoa, 2017) hace referencia a la RSC como la forma de dirigir a una empresa, según los impactos que generan sus acciones sobre los socios, empleados, medioambiente o la comunidad en general. Por lo tanto, la RSC es interna porque cada institución cuenta con recursos humanos, quienes tienen expectativas y objetivos adyacentes a los de la empresa. De similar forma, es externa debido a los diversos sectores relacionados (socios, proveedores, inversionistas, entre otros) tienen sus propias expectativas con respecto a la empresa (ANDI, 2001).

En países donde carecen de una legislación sobre derechos sociales o normas medioambientales, deben centrar sus esfuerzos en la conformación de leyes, donde se posibilite el desarrollo de prácticas socialmente responsables (Vélez \& Cano, 2016). Varios autores coinciden en que las tres principales dimensiones de la RSC son: la económica, área social y medio ambiental. No obstante, en estudios más recientes aparecen dos nuevas dimensiones; una relacionada a los stakeholders y otra a los voluntariados (Arsić et al., 2017) (Ver figura 2).

Dentro de cada dimensión, se distinguen varios conceptos. La dimensión economía contiene: obtención de los máximos beneficios, satisfacer expectativas de los socios, ser líder en el mercado, entre otras; la dimensión social, se compone por: la creación de empleo, respetar para defender los derechos humanos, capacitación a los empleados, colaborar con instituciones públicas y organizaciones benéficas. La dimensión ambiental, analiza aspectos como: la reducción de desperdicios, proteger la biodiversidad y promover el reciclaje (González-Rodríguez et al., 2015). 
Figura 2. Dimensiones de la Responsabilidad Social

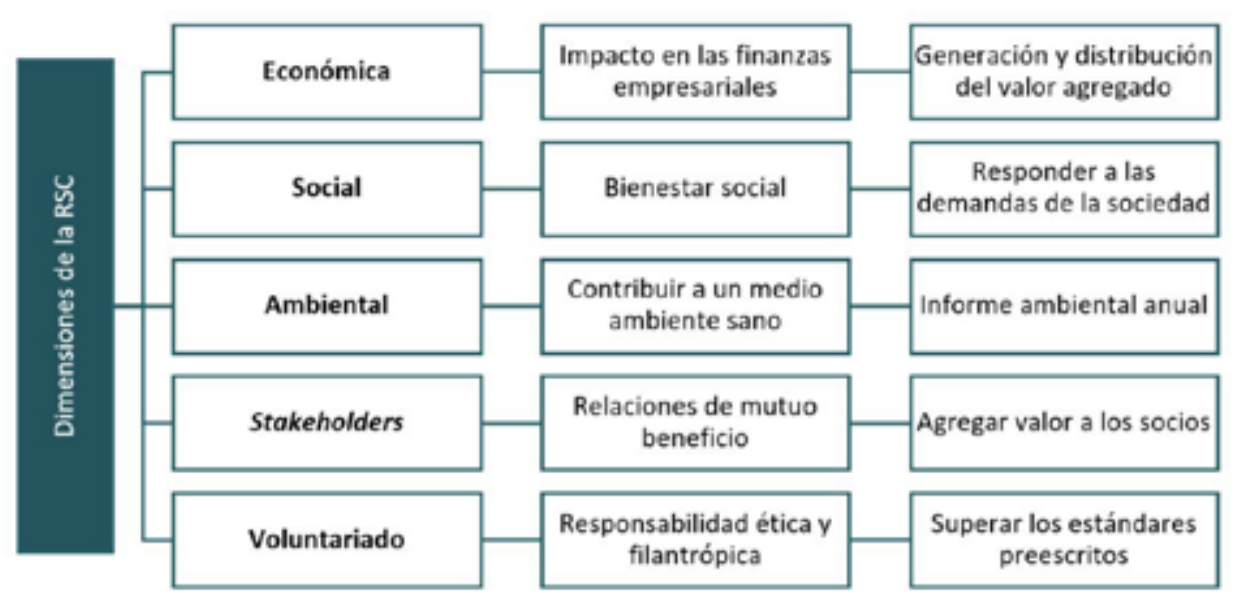

Fuente: Adaptado de Arsić et al. (2017). Las dimensiones más importantes de la RSC.

\section{Imagen corporativa}

El objetivo empresarial es adentrarse en la mente de los consumidores. De ahí, la imagen corporativa está establecida con base a la percepción que tienen los diversos grupos de interés (público interno y externo), sobre las acciones llevadas a cabo por una organización. Dentro del modelo de gestión empresarial, la construcción de una imagen corporativa debe ser un pilar fundamental para el desarrollo de la estrategia. De igual manera, es necesario aplicar una apropiada medición de la imagen corporativa, a través de una evaluación a la percepción que tienen los stakeholders en torno a los factores como la confianza institucional, el aporte a la comunidad o entorno (Pasquel et al., 2016).

De esta forma, gestionar adecuadamente la marca corporativa para garantizar su éxito, involucra analizar, planificar y gestionar los elementos que inciden en la creación de imagen; además, de los aspectos relevantes en la interrelación entre la organización y el público. De acuerdo con Capriotti (2010), dentro del proceso estratégico existen tres 
etapas: el análisis estratégico, la definición del perfil de identidad y la comunicación.

El análisis estratégico de situación toma en cuenta aspectos organizacionales, como: entorno del público e imagen corporativa; todos estos elementos, permiten diagnosticar la situación de la empresa y tomar las decisiones correspondientes. La segunda etapa corresponde a la definición del Perfil de Identidad Corporativa (PIC); este proceso corresponde a la elección de los atributos que forman parte de la cultura y filosofía corporativa. La última etapa, denominada gestión estratégica de la identidad corporativa, menciona que, la comunicación asertiva del PICd, depende del rol que cumplan los comunicadores y de las expectativas creadas alrededor del bien o servicio (Capriotti, 2010).

Figura 2. Gestión estratégica de imagen corporativa

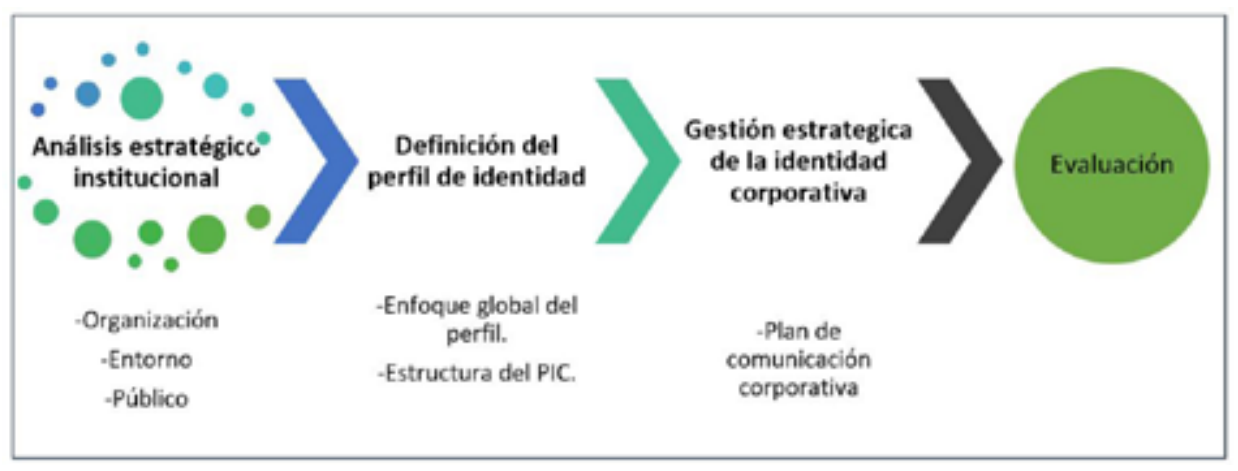

Fuente: Adaptado de Capriotti (2010). Branding corporativo: Gestión estratégica de la identidad.

La reputación de una empresa puede influir positivamente en la decisión de compra de los consumidores; esto a su vez, permite alcanzar una ventaja competitiva sobre sus rivales al considerar la efectiva imagen corporativa que ayuda a expandir las ventas. Por consiguiente, es importante tomar en cuenta la percepción de la imagen corporativa por parte de los empleados, dado que, puede influir significativamente en sus actitudes y en el desempeño (Teng et al., 2000). 
Diversos autores coinciden en que, la RSC influye en la construcción de una imagen corporativa atractiva que permita alcanzar el éxito empresarial (Virvilaite \& Daubaraite, 2011)basing the findings theoretically and empirically. The first part of this article focuses on a theoretical concept of corporate social responsibility. The authors analyze different definitions of corporate social responsibility, review chronological development of this concept and reveal similarities between different definitions. Positive and negative positions towards corporate social responsibility are presented and compared. The second part of this article reveals the theoretical concept of corporate image and the constituting factors. After discussing different definitions of corporate image, concluding remarks are made. The authors of this article discuss the topicality of corporate image management subsequently and name the factors that form corporate image, laying out the basis to manage particular factors. The first two parts of this paper provide a theoretical basis for further discussion. The third part of this article outlines insights for causality between corporate social responsibility and corporate image as well as links between the two. Concluding the reviewed literature and the newest empirical data, it is noted that corporate social responsibility has positive impact on corporate image. Positive impact can be seen through various phenomena: positive consumer attitude, positive word of mouth, etc. Theoretical studies lay the basis for creating a structural model of corporate social responsibility impact on corporate image, which presents the impact corporate social responsibility has on corporate image. In addition to previously discussed theoretical concepts, three factors forming corporate image (corporate marketing communications, corporate social responsibility and consumer demographic characteristics. La imagen constituye una ventaja competitiva de las organizaciones. De tal forma, la imagen corporativa no debe estar constituida únicamente por el logotipo o slogan, sino, debe estar en función a la razón de ser de la empresa y difundir un mensaje claro de confianza y credibilidad hacia los stakeholders (Pasquel et al., 2016). Cabe destacar que, la evaluación organizacional puede ser subjetiva, ya que todos los involucrados tienen diferentes percepciones al momento de evaluar la imagen de una organización y, en muchos casos, dependen de sus propias experiencias (Pasquel et al., 2016). 


\section{Sector cooperativista del Ecuador}

Las cooperativas de ahorro y crédito se encuentran dentro de la clasificación como instituciones partícipes de la Economía popular y solidaria, donde su objetivo principal es el crecimiento individual y colectivo de la comunidad bajo un ambiente social justo y sustentable (SEPS, 2021). Con base a García, Prado, Salazar \& Mendoza (2018) en distintos países latinoamericanos, las Cooperativas de Ahorro y Crédito realizan actividades de intermediación financiera en beneficio de los socios, y están integradas por un conjunto de personas que aportan de forma equitativa al capital social, lo que les permite constituir los recursos necesarios para alcanzar el desarrollo.

Las instituciones financieras, en especial, las organizaciones del sistema económico social y solidario tienen la responsabilidad de rendir cuentas a sus socios y mantener una relación equilibrada entre sociedad, estado y mercado. Por lo tanto, se guía por principios como: la búsqueda del buen vivir y el bien común; la prelación del trabajo sobre el capital; el comercio justo, consumo ético y responsable; la equidad de género; el respeto a la identidad cultural; la responsabilidad ambiental y la distribución equitativa de los excedentes (Ley Orgánica de Economía Popular y Solidaria, 2011).

Las acciones de responsabilidad social se fundamentan como el conjunto de actividades voluntarias para servir a la comunidad. Mediante la Disposición General Segunda de la Ley Orgánica de Economía Popular y Solidaria LOEPS, las organizaciones deben incorporar en sus informes de gestión, el balance social que se define como el instrumento que reúne y sistematiza la información de las áreas económicas, medioambientales y sociales, con el fin de determinar las acciones programadas por las entidades parte de la SEPS para dar cumplimiento a determinados valores y principios establecidos por cada entidad (SEPS, 2017).

En el Ecuador, la Junta de Política de Regulación Monetaria y Financiera es el ente regulador responsable de la formulación de políticas y la regulación monetaria. Esta determina en qué segmentos se ubican las cooperativas, de acuerdo con la Norma para la segmentación de entidades del sector financiero popular y solidario. En el Segmento 1, se encuentran aquellas entidades con un monto de sus activos mayor a $\$$ $80^{\prime} 000.000,00$; en el segmento 2 con un valor mayor a $\$ 20^{\prime} 000.000,00$ 
hasta $80^{\prime} 000.000,00$; segmento 3 un monto superior a $\$ 1^{\prime} 000.000,00$ hasta $\$ 5^{\prime} 000.000 .00$; y en el segmento 5 a las entidades con activos hasta $\$ 1^{\prime} 000.000,00$ así como a las Cajas de Ahorro, bancos comunales y cajas comunales (SEPS, 2015; CUC, 2017).

Las cooperativas de ahorro y crédito activas en la provincia de Tungurahua registradas en la Superintendencia de Economía Popular y Solidaria (SEPS), son las siguientes: en el segmento 1, 8 cooperativas y 1 mutualista; segmento 2, un total de 3 instituciones; segmento 3, 13 cooperativas y a los segmentos 4 y 5 perteneces 28 entidades. En conjunto, los activos de las cooperativas de ahorro y crédito igualan los 13.783 millones de dólares, distribuidos de la siguiente manera: el segmento 1 superan los 10.360 millones de dólares; el segmento 2, 1886 millones; los activos de las cooperativas de ahorro del segmento 3 y 4, 964 millones y 467 millones respectivamente; y el segmento 5 posee 104 millones de dólares en activos (Asobanca, 2019).

Durante los últimos años el sector cooperativista se ha fortalecido y ha alcanzado resultados importantes, mediante la otorgación de créditos a diversos sectores del Ecuador. Es así que, el $45 \%$ del total de depositantes del segmento 1 se concentran en las provincias de Pichincha, Azuay y Tungurahua y presentan regularmente información estadística al organismo regulador correspondiente (CORDES, 2018). La percepción de la RSC de las empresas, en especial de las instituciones financieras, son importantes, no solo al momento de evaluar los riesgos de los inversionistas y socios, sino también para conseguir la confianza del público en el sistema financiero (Jizi et al., 2014). 


\section{Metodología}

La presente investigación se realizó bajo un diseño no experimental, debido a que no se modificó ni se alteró las variables de investigación. Se analizó la relación entre la responsabilidad social y la imagen corporativa en el sector cooperativista de los segmentos 3 y 4 de la provincia de Tungurahua bajo condiciones preexistentes (Sampieri et al., 2014). El enfoque de la investigación fue cuantitativo con un alcance correlacional en vista que, mediante la recolección de datos numéricos y el procesamiento estadístico de datos se analizó el criterio de los gerentes de las cooperativas con relación a la responsabilidad social e imagen corporativa con el objetivo de determinar si existe o no, una relación positiva entre las variables.

Se utilizó la técnica bibliográfica para la construcción teórica respecto a las variables de investigación responsabilidad social e imagen corporativa. Mediante la técnica de campo se llevó a cabo el levantamiento de información de los participantes extraídos de la base de datos de las cooperativas registradas en la SEPS, tomando en cuenta a aquellas cooperativas de los segmentos 3 y 4 .

El instrumento para la recopilación de datos constó de 32 elementos estructurados en 4 secciones: a) imagen corporativa, b) socios externos, c) socios internos y d) inversionistas. La escala de Likert de 5 puntos fue utilizada para valorar la percepción de los gerentes organizacionales, donde 1 =Totalmente en desacuerdo; $2=$ En desacuerdo; $3=$ Indiferente; 4= De acuerdo; $5=$ Totalmente acuerdo (Quero, 2010). Se aplicó el coeficiente de fiabilidad de Cronbach para obtener el grado de confiabilidad de la encuesta como se señaló en la ecuación 1.

$$
\alpha=\frac{K}{K-1}\left(\frac{\sum \frac{K}{i=1} \sigma_{Y_{i}}^{2}}{\sigma \frac{2}{X}}\right)
$$

Ecuación 1. Fórmula de alfa Cronbach 
Ante ello, se obtuvo un valor $\alpha=, 964$ (96\%), lo que significó que tiene una alta confiabilidad. Ante ello, se obtuvo un valor $\alpha=, 964$ (96\%), lo que significó que tiene una alta confiabilidad (ver tabla 1).

Tabla 1. Confiabilidad del instrumento

\section{Alfa de Cronbach Número de elementos}

Fuente: Adaptado de los datos obtenidos del procesamiento estadístico en el software SPSS.

Como población de análisis se tomó a las Cooperativas de Ahorro y Crédito de los segmentos 3 y 4 de la provincia de Tungurahua. Se utilizó un muestreo no probabilístico por conveniencia para la selección de la muestra, ya que, este tipo de muestreo permite a los investigadores establecer criterios de selección con base a características propias del estudio o según se estime conveniente (Muñoz, 2018). La base de datos consta de 37 cooperativas de ahorro y crédito, seleccionadas por los criterios de: a) aporte a la comunidad, b) predisposición en la presentación de información sobre la responsabilidad social. En este caso, se excluyó a las instituciones que no presentaron su información de balance social y están inactivas según la SEPS. Por prevención y acatar el protocolo de bioseguridad, las encuestas se ejecutaron, a través de la plataforma Google drive (encuestas digitales) (ver tabla 2). 
Tabla 2. Ficha técnica de investigación

\begin{tabular}{|c|c|}
\hline Parámetro & Descripción \\
\hline $\begin{array}{l}\text { Muestra de } \\
\text { estudio }\end{array}$ & $\begin{array}{l}37 \text { Cooperativas de Ahorro y } \\
\text { Crédito de los segmentos } 3 \text { y } 4\end{array}$ \\
\hline Entorno & $\begin{array}{l}\text { Provincia de Tungurahua-Am- } \\
\text { bato }\end{array}$ \\
\hline $\begin{array}{l}\text { Método de } \\
\text { captación }\end{array}$ & Encuesta \\
\hline Procedimiento & $\begin{array}{l}\text { Muestreo de tipo no probabi- } \\
\text { lístico }\end{array}$ \\
\hline $\begin{array}{l}\text { Nivel de con- } \\
\text { fianza }\end{array}$ & $95 \%$ \\
\hline Nivel de error & $5 \%$ \\
\hline $\begin{array}{l}\text { Cuestionarios } \\
\text { validados }\end{array}$ & 37 \\
\hline
\end{tabular}

Fuente: Elaboración propia. 


\section{Resultados}

De acuerdo con el análisis del perfil sociodemográfico de los gerentes, se determinó que el $62 \%$ de los encuestados son mujeres y el $38 \%$ son hombres. Esto ha evidenciado la inclusión de las mujeres en los segmentos 3 y 4 de las cooperativas de ahorro y crédito. Además, se demostró la presencia de talento joven dentro de las organizaciones, ya que, el rango de edad de los gerentes se encuentra en el rango de 25 y 45 años. De ellos, el $65 \%$ posee un título de tercer nivel, el 27\% culminó la secundaria, y apenas el $8 \%$ ha seguido un estudio de posgrado.

El coeficiente de correlación de Spearman demostró el nivel de asociación entre los componentes de los factores de responsabilidad social. Dentro del factor imagen, a un nivel de $99 \%$ de significancia, se evidenció la importancia de proporcionar información sobre las actividades que realizan las cooperativas en favor del respeto a los derechos humanos (0.504). De igual manera, existe un grado de relación similar entre contar con una normativa de protección a los socios para seguir un código de ética empresarial (0.528); finalmente, la relación entre los donativos a instituciones benéficas está directamente relacionado con el esfuerzo para proteger el medio ambiente (0.594) y contribuir a campañas públicas (0.448) (ver tabla 3). 
Tabla 3. Correlación factor imagen

\begin{tabular}{|c|c|c|c|c|c|c|c|c|c|}
\hline Factor Imagen & 1 & 2 & 3 & 4 & 5 & 6 & 7 & 8 & 9 \\
\hline $\begin{array}{l}\text { 1. La cooperativa sigue } \\
\text { la normativa de pro- } \\
\text { tección a los socios }\end{array}$ & 1 & & & & & & & & \\
\hline $\begin{array}{l}\text { 2. Posee un departa- } \\
\text { mento específico de } \\
\text { responsabilidad social }\end{array}$ & ,426* & 1 & & & & & & & \\
\hline $\begin{array}{l}\text { 3. Proporciona infor- } \\
\text { mación constante al } \\
\text { público sobre las acti- } \\
\text { vidades que realiza la } \\
\text { cooperativa }\end{array}$ & ,149 &,- 051 & 1 & & & & & & \\
\hline $\begin{array}{l}\text { 4. La institución partici- } \\
\text { pa en obras de caridad } \\
\text { o realiza donativos a } \\
\text { instituciones benéficas }\end{array}$ & ,382* &, $504^{* *}$ & ,148 & 1 & & & & & \\
\hline $\begin{array}{l}\text { 5. Respeta los dere- } \\
\text { chos humanos }\end{array}$ & ,223 &,- 252 &, $520^{* *}$ & ,042 & 1 & & & & \\
\hline $\begin{array}{l}\text { 6. Contribuye a cam- } \\
\text { pañas públicas (ej. An- } \\
\text { tidrogas, antitabaco, } \\
\text { otro) }\end{array}$ &, $356^{*}$ & ,499** & ,309 & , 448* &, $346^{*}$ & 1 & & & \\
\hline $\begin{array}{l}\text { 7. Realiza esfuerzos } \\
\text { para proteger el me- } \\
\text { dio ambiente }\end{array}$ & ,430* & ,281 &,- 016 &, $594^{* *}$ & , 182 & ,296 & 1 & & \\
\hline $\begin{array}{l}\text { 8. Contribuye en el } \\
\text { desarrollo de la comu- } \\
\text { nidad }\end{array}$ &, $340^{*}$ & ,115 &,- 054 & ,058 & ,399* &, $422^{* *}$ &, 091 & 1 & \\
\hline $\begin{array}{l}\text { 9. Posee un código de } \\
\text { ética empresarial }\end{array}$ & ,528* &, $429^{* *}$ & ,241 & ,243 & ,237 &, $348^{*}$ &, $461^{* *}$ &, 049 & 1 \\
\hline \multicolumn{10}{|c|}{ ** La correlación es significativa al nivel 0,01 (bilateral). } \\
\hline * La correlación es significati & a al niv & el 0,05 & (bilater & & & & & & \\
\hline
\end{tabular}


El factor socios externos demostró un alto grado de significancia entre todos sus componentes (valor $p<0,01$ ). Principalmente, la categoría brindar facilidades de pago a los socios se relacionó con la innovación en los productos (0.733) y variedad de servicios financieros $(0.461)$. Por otro lado, fijar las tasas de interés con base a las tasas referenciales del BCE afecta a la variedad de servicios que ofrecen las cooperativas (0.516). Además, los pagos de productos financieros son facilitados por parte de los socios (ver tabla 4).

Tabla 4.Correlación factor socios externos

\begin{tabular}{|c|c|c|c|c|}
\hline Factor Socios externos & 10 & 11 & 12 & 13 \\
\hline $\begin{array}{l}\text { 10. Las tasas de interés son fijadas con } \\
\text { base a las tasas referenciales del BCE }\end{array}$ & 1 & & & \\
\hline $\begin{array}{l}\text { 11. Ofrece variedad de productos / servi- } \\
\text { cios financieros }\end{array}$ &, $516^{* *}$ & 1 & & \\
\hline $\begin{array}{l}\text { 12. Existe innovación en la presentación } \\
\text { de productos/servicios financieros }\end{array}$ &, $461^{* *}$ & $600^{* *}$ & 1 & \\
\hline $\begin{array}{l}\text { 13. Brinda facilidades de pago de los pro- } \\
\text { ductos / servicios a los socios }\end{array}$ & ,622** &, $550^{* *}$ &, $733^{* *}$ & 1 \\
\hline \multicolumn{5}{|c|}{ ** La correlación es significativa al nivel 0,01 (bilateral). } \\
\hline \multicolumn{5}{|c|}{ * La correlación es significativa al nivel 0,05 (bilateral). } \\
\hline
\end{tabular}

El factor socios internos con relación a los empleados, evidenció un alto nivel de relación entre la presencia de un manual de funciones y brindar oportunidades de crecimiento al personal (0.777). Esto significa que, las variables contienen características similares que les permiten relacionarse significativamente (99\%). Del mismo modo, se demostró relación entre: los empleados y los canales de retroalimentación, pues, estuvieron ligados a contar con espacio físico suficiente para llevar a cabo sus actividades (0.694) (ver tabla 5). 
Ante lo mencionado, se interpreta que, una efectiva imagen corporativa denota que la organización es administrada por personas responsables y comprometidas con su manejo. Con base a este argumento, Lopéz (2007) menciona que, si las condiciones de trabajo son atractivas, existe compromiso e integración con los objetivos organizacionales por parte de los empleados. No obstante, se presentó una relación entre la inversión en desarrollo y las oportunidades de crecimiento empresarial (671), lo cual, indica la importancia del ambiente laboral para los empleados y la organización en general. 


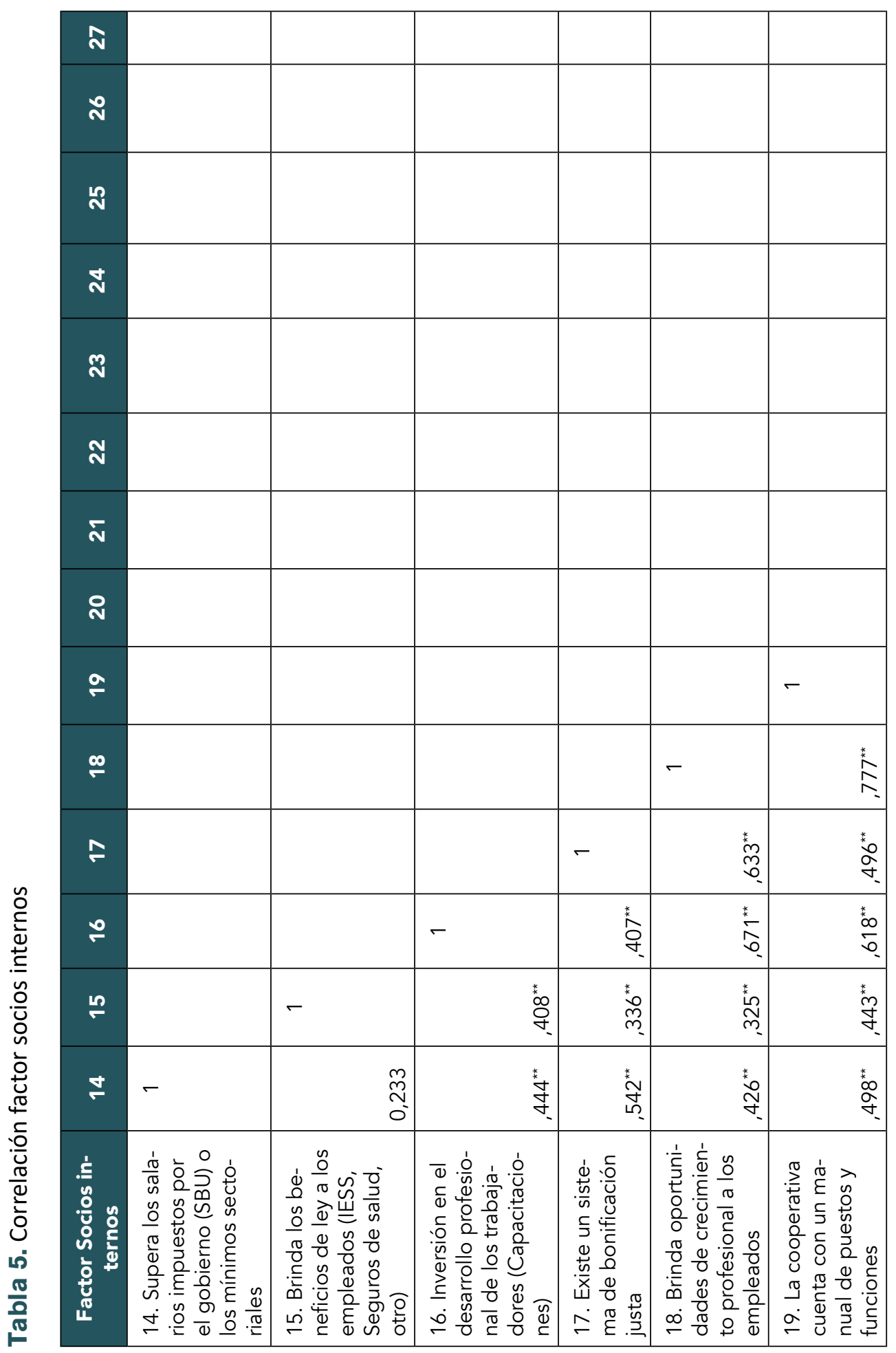

ลู 


\begin{tabular}{|c|c|c|c|c|c|c|c|c|}
\hline & & & & & & & - & \\
\hline & & & & & & - & in & \\
\hline & & & & & - & 。్ & مo & \\
\hline & & & & - & 雚 & 吕 & $\begin{array}{l}\infty \\
0 \\
0\end{array}$ & \\
\hline & & & - & 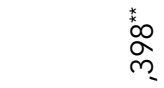 & 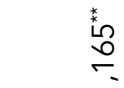 & ô & స్య & \\
\hline & & - & $\begin{array}{l}\text { an } \\
\text { on. }\end{array}$ & $\underset{\sim}{\sim}$ & 年 & 量 & 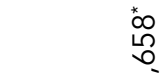 & \\
\hline & - & $\begin{array}{l}\text { yे } \\
\text { t }\end{array}$ & 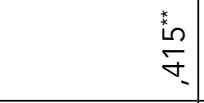 & 施 & 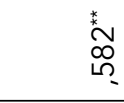 & 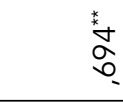 & m. & \\
\hline - & $\begin{array}{l}g \\
g \\
0\end{array}$ & $\begin{array}{l}\frac{n}{2} \\
0 \\
0\end{array}$ & 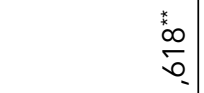 & 悉 & స్ల్ల & ${ }_{\substack{\infty \\
\infty}}^{\infty}$ & 㹲 & \\
\hline 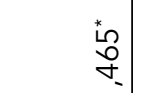 & $\begin{array}{l}\infty \\
\vdots \\
0\end{array}$ & 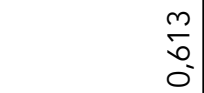 & 容 & חم: & 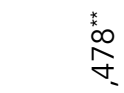 & 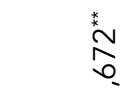 & مn & \\
\hline $\begin{array}{l}\text { y } \\
\text { hn }\end{array}$ & $\begin{array}{l}\frac{n}{0} \\
0 \\
0\end{array}$ & 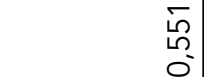 & in & 㝵 & f & 总 & to & \\
\hline 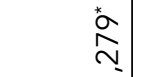 & $\begin{array}{l}\text { o. } \\
\text { on }\end{array}$ & f & $\tilde{Z}$ & $\stackrel{d}{\sigma_{N}^{\alpha}}$ & 每 & 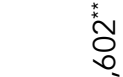 & 㤐 & \\
\hline 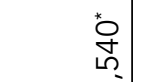 & $\stackrel{m}{g}_{0}^{\frac{m}{0}}$ & 恿 & 莡 & $\stackrel{\infty}{\sim}$ & : & 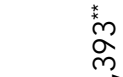 & $\overline{\grave{m}}$ & Бे. \\
\hline 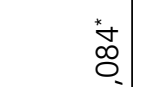 & $\begin{array}{c}\infty \\
m \\
m \\
0\end{array}$ & $\stackrel{\widehat{N}}{\tilde{N}_{0}}$ & & 용 & 来 & $\stackrel{f}{g}$ & $\stackrel{\infty}{\sigma}$ & \\
\hline $\begin{array}{l}\text { to } \\
\text { n }\end{array}$ & $\begin{array}{l}0 \\
y \\
0\end{array}$ & (1) & g & 哭 & 孟 & 莒 & in & \\
\hline 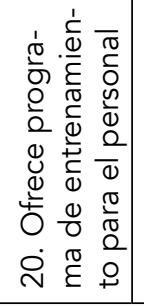 & 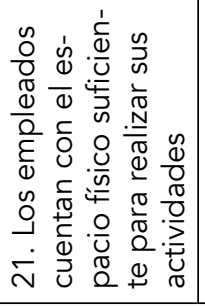 & 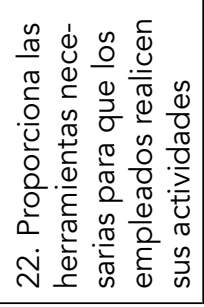 & 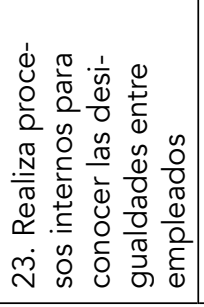 & 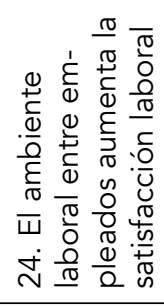 & 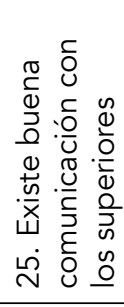 & 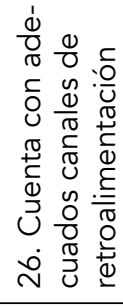 & 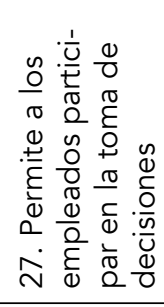 & 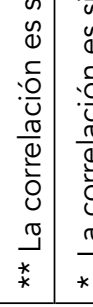 \\
\hline
\end{tabular}


La información es un recurso fundamental dentro de cualquier tipo de organización, dado que, proporciona herramientas de planificación y estrategias de negocios prescindibles para la toma de decisiones de los gerentes y acreedores de información (Arribas, 2000; Marrero, 2013). Particularmente, para el factor de inversionistas fue fundamental que las cooperativas brinden información sobre las actividades que realizan y los planes de inversión a futuro. Es decir, se identificó una correlación de (0.881) entre estas variables a un nivel de significancia del 95\%. Asimismo, existió una relación del (0.439) entre la capacidad para atraer nuevos inversionistas e informar sobre las decisiones de inversión (ver tabla 6).

Tabla 6. Correlación factor inversionistas

\begin{tabular}{|l|l|l|l|l|l|}
\hline \multicolumn{1}{|c|}{$\begin{array}{c}\text { Factor } \\
\text { Inversionistas }\end{array}$} & \multicolumn{1}{c|}{$\mathbf{2 8}$} & $\mathbf{2 9}$ & $\mathbf{3 0}$ & $\mathbf{3 1}$ & $\mathbf{3 2}$ \\
\hline $\begin{array}{l}\text { 28. La cooperativa } \\
\text { es rentable y gene- } \\
\text { ra beneficios a sus } \\
\text { socios }\end{array}$ & 1 & & & & \\
\hline $\begin{array}{l}\text { 29. La cooperativa } \\
\text { es capaz de atraer a } \\
\text { nuevos inversionis- } \\
\text { tas y socios }\end{array}$ & 0,128 & 1 & 1 & & \\
\hline $\begin{array}{l}\text { 30. Informa a los so- } \\
\text { cios sobre las deci- } \\
\text { siones de inversión }\end{array}$ &, $371^{*}$ &, $439^{*}$ & 1 & & \\
\hline $\begin{array}{l}31 . \text { Informa a los } \\
\text { socios sobre los } \\
\text { proyectos futuros }\end{array}$ &, $350^{*}$ &, $410^{*}$ &, $881^{*}$ & 1 & 1 \\
\hline $\begin{array}{l}32 . \text { Informa sobre la } \\
\text { implementación de } \\
\text { políticas empresa- } \\
\text { riales }\end{array}$ & 0,173 & 0,594 & 0,671 & 0,599 & 1 \\
\hline ** La correlación es significativa al nivel 0,01 (bilateral). \\
\hline * La correlación es significativa al nivel 0,05 (bilateral). \\
\hline
\end{tabular}


El modelo de regresión múltiple validó la influencia de las variables predictivas, pues, se comprobó los modelos ajustados entre imagen corporativa, socios externos, socios internos e inversionistas. Se evidenció un valor $\mathrm{R}$ cercano a 1 en el factor socios internos $(0,771)$ y un coeficiente de determinación $\left(R^{\wedge} 2\right)$ explicó el $59 \%$ de variabilidad positiva, como lo señaló el coeficiente de Durbin Watson al ser mayor que 2, entre los socios internos y la imagen corporativa. Sin embargo, los demás factores no se ajustan al modelo, puesto que, presentaron valores $\mathrm{R}^{\wedge} 2$ por debajo de 0,4 (ver tabla 7). Por último, los factores imagen y socios internos destacaron como determinantes de influencia de la responsabilidad social e imagen corporativa positiva, puesto que, se denotó la importancia de cumplir con las necesidades y expectativas de los grupos de interés.

Tabla 7. Modelo de regresión múltiple

\begin{tabular}{|l|r|r|r|r|r|}
\hline \multicolumn{1}{|c|}{ Modelo } & \multicolumn{1}{|c|}{$\mathbf{R}$} & $\mathbf{R}$ cuadrado & $\begin{array}{c}\text { R cuadrado } \\
\text { corregida }\end{array}$ & $\begin{array}{c}\text { Error típ. } \\
\text { de la esti- } \\
\text { mación }\end{array}$ & \multicolumn{1}{c|}{$\begin{array}{c}\text { Durbin-Wat- } \\
\text { son }\end{array}$} \\
\hline $\begin{array}{l}\text { Imagen } \\
\text { corpora- } \\
\text { tiva }\end{array}$ &, $590^{\mathrm{a}}$ &, 348 &, 131 & 1,269 & 1,763 \\
\hline $\begin{array}{l}\text { Socios } \\
\text { externos }\end{array}$ &, $319^{\mathrm{a}}$ &, 102 &,- 010 & 1,368 & 1,682 \\
\hline $\begin{array}{l}\text { Socios } \\
\text { internos }\end{array}$ &, $771^{\mathrm{a}}$ &, 594 &, 335 & 1,110 & 2,565 \\
\hline $\begin{array}{l}\text { Inversio- } \\
\text { nistas }\end{array}$ &, $461^{\mathrm{a}}$ &, 212 &, 085 & 1,302 & 1,974 \\
\hline
\end{tabular}

Fuente: Elaboración propia

Con el análisis de coeficientes beta se pudo determinar que dentro del eje imagen, proporcionar información sobre las actividades que realiza la cooperativa (0.006) tiene una relación directa con la construcción de una imagen corporativa. Es decir, los resultados revelaron que los 
gerentes constantemente proporcionan información a su público sobre las actividades que realizan y lo consideran un factor imprescindible para la construcción de una imagen empresarial.

El eje socios externos reveló que las instituciones no brindan facilidades de pago, a su vez mostraron que las tasas de interés son altas. Sin embargo, las instituciones sí ofertan variedad de productos y servicios financieros, lo cual, las expone a mantener una buena reputación frente a las demás instituciones (0.084). Por otra parte, el factor socios internos expuso que, las cooperativas construyen una imagen corporativa positiva a través de una buena comunicación entre el personal operativo y la gerencia (0.001); también, se realizan procesos para conocer las desigualdades entre empleados (0.036) y brindarles los beneficios de ley (0.037).

Los socios de las cooperativas de ahorro y crédito relacionan directamente la imagen corporativa con la proporción de información sobre los nuevos proyectos, inversiones y planes futuros, que les brinde seguridad sobre la rentabilidad y generación de beneficios. El factor inversionistas denotó la información que proporcionan las cooperativas a los socios sobre las decisiones de inversión (ver tabla 8). En síntesis, las cooperativas no favorecen a todos los grupos de interés de la organización según las pautas de responsabilidad social, pues, se descuida los factores de imagen, socios externos y a los inversionistas que también son factores clave para las organizaciones. Las instituciones han sentido la obligación y responsabilidad de cumplir con sus empleados, y estos se tornan como el principal eje para la construcción de una imagen corporativa positiva. 
Tabla 8. Análisis de coeficientes Beta

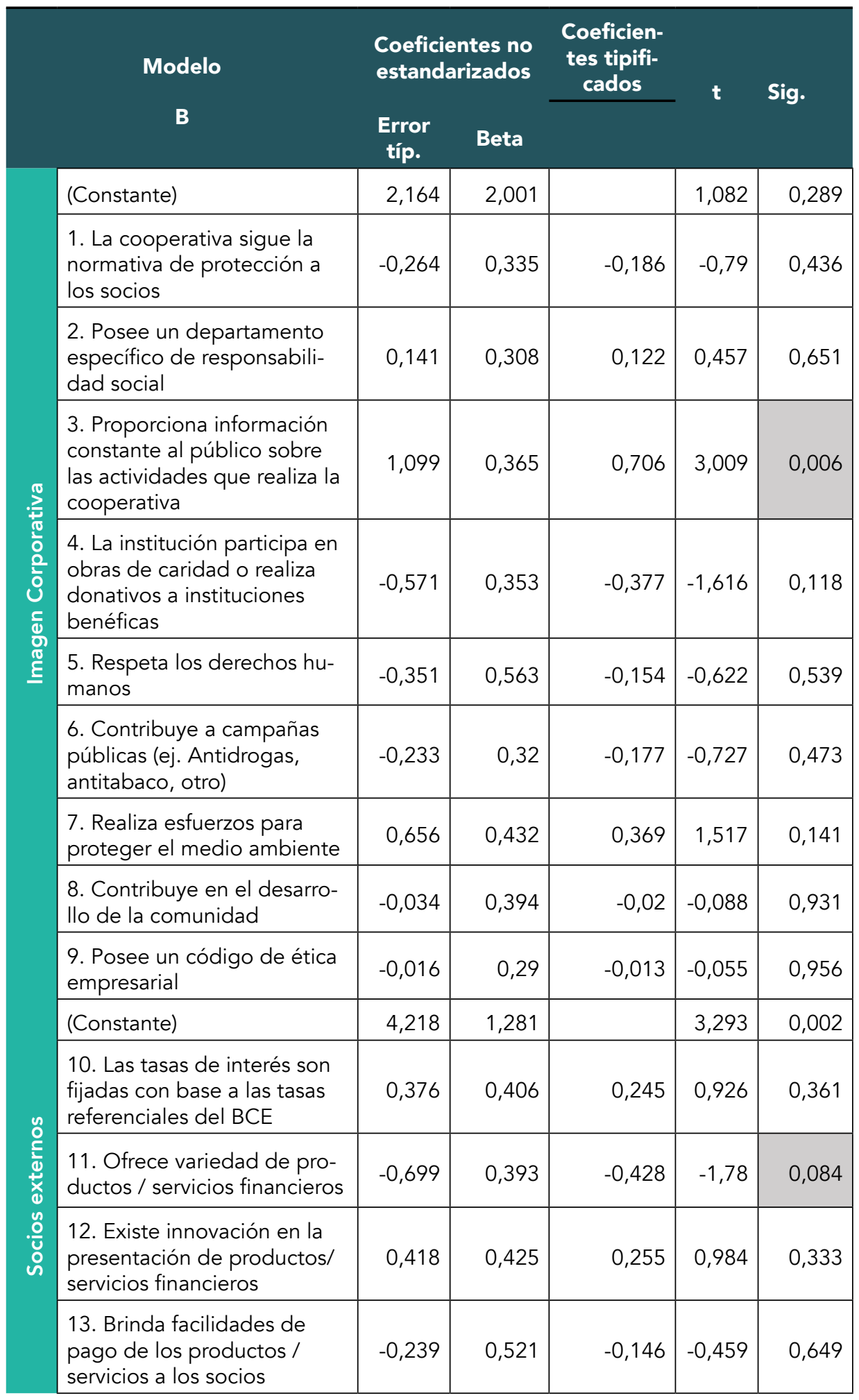




\begin{tabular}{|c|c|c|c|c|c|}
\hline (Constante) & 3,402 & 1,252 & & 2,718 & 0,013 \\
\hline $\begin{array}{l}\text { 14. Supera los salarios im- } \\
\text { puestos por el gobierno } \\
\text { (SBU) o los mínimos secto- } \\
\text { riales }\end{array}$ & 0,407 & 0,345 & 0,275 & 1,181 & 0,25 \\
\hline $\begin{array}{l}\text { 15. Brinda los beneficios de } \\
\text { ley a los empleados (IESS, } \\
\text { Seguros de salud, otro) }\end{array}$ & 0,69 & 0,312 & 0,516 & 2,214 & 0,037 \\
\hline $\begin{array}{l}\text { 16. Inversión en el desarrollo } \\
\text { profesional de los trabajado- } \\
\text { res (Capacitaciones) }\end{array}$ & $-0,963$ & 0,302 & $-0,772$ & $-3,192$ & 0,004 \\
\hline $\begin{array}{l}\text { 17. Existe un sistema de } \\
\text { bonificación justa }\end{array}$ & 0,274 & 0,484 & 0,183 & 0,566 & 0,577 \\
\hline $\begin{array}{l}\text { 18. Brinda oportunidades de } \\
\text { crecimiento profesional a los } \\
\text { empleados }\end{array}$ & 0,467 & 0,417 & 0,337 & 1,119 & 0,275 \\
\hline $\begin{array}{l}\text { 19. La cooperativa cuenta } \\
\text { con un manual de puestos y } \\
\text { funciones }\end{array}$ & 0,287 & 0,407 & 0,218 & 0,705 & 0,488 \\
\hline $\begin{array}{l}\text { 20. Ofrece programa de en- } \\
\text { trenamiento para el personal }\end{array}$ & 0,27 & 0,364 & 0,196 & 0,742 & 0,466 \\
\hline $\begin{array}{l}\text { 21. Los empleados cuentan } \\
\text { con el espacio físico sufi- } \\
\text { ciente para realizar sus acti- } \\
\text { vidades }\end{array}$ & 0,216 & 0,437 & 0,138 & 0,495 & 0,625 \\
\hline $\begin{array}{l}\text { 22. Proporciona las herra- } \\
\text { mientas necesarias para que } \\
\text { los empleados realicen sus } \\
\text { actividades }\end{array}$ & $-0,381$ & 0,435 & $-0,23$ & $-0,874$ & 0,391 \\
\hline $\begin{array}{l}\text { 23. Realiza procesos internos } \\
\text { para conocer las desigualda- } \\
\text { des entre empleados }\end{array}$ & $-0,763$ & 0,342 & $-0,669$ & $-2,235$ & 0,036 \\
\hline $\begin{array}{l}\text { 24. El ambiente laboral entre } \\
\text { empleados aumenta la satis- } \\
\text { facción laboral }\end{array}$ & $-0,492$ & 0,479 & $-0,307$ & $-1,028$ & 0,315 \\
\hline $\begin{array}{l}25 \text {. Existe buena comunica- } \\
\text { ción con los superiores }\end{array}$ & $-1,662$ & 0,442 & $-1,219$ & $-3,759$ & 0,001 \\
\hline $\begin{array}{l}\text { 26. Cuenta con adecuados } \\
\text { canales de retroalimentación }\end{array}$ & 0,612 & 0,49 & 0,388 & 1,248 & 0,225 \\
\hline $\begin{array}{l}\text { 27. Permite a los empleados } \\
\text { participar en la toma de } \\
\text { decisiones }\end{array}$ & 1,024 & 0,364 & 0,754 & 2,811 & 0,01 \\
\hline
\end{tabular}




\begin{tabular}{|l|r|r|r|r|r|}
\hline (Constante) & 2,213 & 1,266 & & 1,748 & 0,09 \\
\hline $\begin{array}{l}\text { 28. La cooperativa es renta- } \\
\text { ble y genera beneficios a sus } \\
\text { inversionistas }\end{array}$ & $-0,279$ & 0,199 & $-0,238$ & $-1,401$ & 0,171 \\
\hline $\begin{array}{l}\text { 29. La cooperativa es capaz } \\
\text { de atraer a nuevos inversio- } \\
\text { nistas }\end{array}$ & $-0,064$ & 0,368 & $-0,04$ & $-0,174$ & 0,863 \\
\hline $\begin{array}{l}\text { 30. Informa a los socios } \\
\text { sobre las decisiones de in- } \\
\text { versión }\end{array}$ & 1,302 & 0,604 & 0,869 & 2,156 & 0,039 \\
\hline $\begin{array}{l}31 . \text { Informa a los socios so- } \\
\text { bre los proyectos futuros }\end{array}$ & $-0,423$ & 0,474 & $-0,31$ & $-0,892$ & 0,379 \\
\hline $\begin{array}{l}\text { 32. Informa sobre la imple- } \\
\text { mentación de políticas em- } \\
\text { presariales }\end{array}$ & $-0,265$ & 0,464 & $-0,167$ & $-0,572$ & 0,571 \\
\hline
\end{tabular}

Nota. Las cifras marcadas muestran las causas relevantes de incidencia según las dimensiones.

El análisis se varianza ANOVA determinó las diferencias significativas entre los valores medios de las variables. A través del análisis del valor $p$, se obtuvo el nivel de significancia de los factores de responsabilidad social e imagen corporativa. En este sentido, se concluye que no existió una diferencia significativa entre los componentes de responsabilidad social; imagen, socios externos e inversionistas con la construcción de imagen corporativa positiva, puesto que presentaron valores $>0,05$, por lo tanto, se descartó el cuadro de hipótesis de estas variables. Por otro lado, el factor socios internos sí posee un nivel de significancia aceptable $(0,039)$, que permite validar la hipótesis, la satisfacción de las necesidades de los empleados contribuye a la construcción de una imagen corporativa positiva.

De acuerdo con Harriague, Kergaravat, \& Santangelo (2012), en el estudio sobre la relación entre la responsabilidad social y la imagen corporativa encontraron que los consumidores consideran a las acciones de responsabilidad social como medidas publicitarias para mejorar su imagen. Por otro lado, Garcia \& Llorente (2009) en el estudio sobre la responsabilidad social como estrategia para conseguir imagen y reputación consideran que el desarrollo de una actividad social repercute directamente a las actividades económicas de las organizaciones. Basado 
en este argumento se determina que las organizaciones consideran a los empleados como un pilar fundamental para su organización, por lo tanto, aplica las medidas para el adecuado funcionamiento del personal (ver tabla 9).

Tabla 9. Cuadro de hipótesis según ANOVA

\begin{tabular}{|l|r|r|c|}
\hline \multicolumn{1}{|c|}{ Marco hipotético } & $F$ & Sig. & $\begin{array}{c}\text { Validación de } \\
\text { criterio }\end{array}$ \\
\hline $\begin{array}{l}\text { Imagen } \rightarrow \text { imagen } \\
\text { corporativa }\end{array}$ & 1,604 &, $164^{\mathrm{b}}$ & Se descartó \\
\hline $\begin{array}{l}\text { Socios externos } \rightarrow \\
\text { imagen corporativa }\end{array}$ &, 908 &, $471^{\mathrm{b}}$ & Se descartó \\
\hline $\begin{array}{l}\text { Socios internos } \rightarrow \\
\text { imagen corporativa }\end{array}$ & 2,298 &, $039^{\mathrm{b}}$ & Se comprobó \\
\hline $\begin{array}{l}\text { Inversionistas } \rightarrow \\
\text { imagen corporativa }\end{array}$ & 1,672 &, $171^{\mathrm{b}}$ & Se descartó \\
\hline
\end{tabular}




\section{Conclusiones}

La responsabilidad social aborda temas que van más allá del crecimiento organizacional, pues, las instituciones deben ser conscientes del impacto de sus acciones en el ambiente y en la comunidad. Para dar cumplimiento a la responsabilidad social fue necesario tomar en cuenta las dimensiones que influyen en ellas, como son: la dimensión económica, social, ambiental, stakeholders y voluntariado.

Los factores imagen, socios externos e internos y los inversionistas no son considerados prioritarios por las cooperativas, por lo tanto, no influye significativamente en la construcción de una imagen corporativa positiva. Sin embargo, si se desea posicionar a las organizaciones cooperativistas para atraer socios inversionistas se debe construir una imagen corporativa positiva, esto se lo consigue al momento que se genera satisfacción en las necesidades de los grupos de interés de las cooperativas.

Las cooperativas del segmento 3 y 4 de la provincia de Tungurahua, aplican su compromiso y responsabilidad con sus socios internos. Esto se encuentra asociado a los principios de la economía popular y solidaria, que enuncia que las cooperativas de ahorro y crédito deben ser socialmente responsables con sus stakeholders. Ante este análisis, se evidenció que las instituciones descuidan su factor de imagen corporativa externa, ya que su compromiso no ha sido el adecuado según la perspectiva gerencial.

La creación de imagen corporativa de las instituciones de los segmentos 3 y 4 a diferencia de las instituciones posicionadas del segmento 1 , han reflejado cierto grado de compromiso con su entorno externo. No obstante, estos factores permiten reconocer y mejorar las deficiencias de las organizaciones, puesto que, el retribuir un margen de beneficio hacia la sociedad mediante la RSC contribuye a la construcción de una imagen corporativa positiva y efectiva. 


\section{Referencias}

Alberto, H., \& Peñaloza, B. (2021). Impacto de la normalización de la política monetaria de la FED en el acceso al crédito de las empresas latinoamericanas. ECONÓMICAS CUC, 42(1), 34-53.

ANDI. (2001). Manual De Balance Social.

Arribas, A. (2000). Comunicación en la empresa. La importancia de la información interna en la empresa. Revista Latina de Comunicación Social, 3(27).

Arsić, S., Stojanović, A., \& Mihajlović, I. (2017). The Most Important Dimensions of Corporate Social Responsibility. Proceedings of the International May Conference on Strategic Management, 318-336.

Asobanca. (2019). Informe Técnico: Bancos Vs . Asociación de Bancos del Ecuador, 2, 1-44.

Awan, H., Hayat, S., \& Faiz, R. (2018). Antecedents and consequences of corporate image: Conventional and islamic banks. RAE Revista de Administracao de Empresas, 58(4), 418-432. https://doi. org/10.1590/S0034-759020180407

Capriotti, P. (2010). Branding corporativo. Gestión estratégica de la identidad corporativa. Comunicación, 0(27), 15-22.

Cardona, A. (2017, July). La historia de casi 100 años de la responsabilidad social empresarial. La República.

COAC Ambato. (2020). Memoria anual 2019.

Coba, E., Díaz, J., Zurita, E., \& Proaño, P. (2017). La responsabilidad social empresarial en las empresas del Ecuador. Un test de relación con la imagen corporativa y desempeño financiero. Ingeniería Industrial. Actualidad y Nuevas Tendencias Año, 5(18), 26.

CORDES. (2018). Análisis sobre el sector de cooperativas de ahorro y crédito en el Ecuador. 
Correa, J. (2007). Evolución histórica de los conceptos de Responsabilidad Social Empresarial y Balance Social. Semestre Económico, 10(20), 87-102.

CUC. (2017). Catálogo único de cuentas. Sector financiero

García, K., Prado, E., Salazar, R., \& Mendoza, J. (2018). Cooperativas de Ahorro y Crédito del Ecuador y su incidenciaen la conformación del Capital Social (2012-2016). Revista Espacios, 39(28), 1-6.

Garcia, M., \& Llorente, C. (2009). La Responsabilidad Social Corporativa: Una Estrategia para conseguir Imagen y Reputación. Revista ICONO, 13(1697-8293), 95-124.

González-Rodríguez, M. R., Díaz-Fernández, M. C., \& Simonetti, B. (2015). The social, economic and environmental dimensions of corporate social responsibility: The role played by consumers and potential entrepreneurs. International Business Review, 24(5), 836-848. https:// doi.org/10.1016/j.ibusrev.2015.03.002

Harriague, D., Kergaravat, V., \& Santangelo, N. (2012). Responsabilidad Social Empresarial y la Imagen Corporativa.

Jizi, M., Salamanca, A., Dixon, R., \& Stratling, R. (2014). Corporate Governance and Corporate Social Responsibility Disclosure: Evidence from the US Banking Sector. Journal of Business Ethics, 2(2), 210-222.

Ley orgánica de economía popular y solidaria, (2011).

Lopéz, E. (2007). Sobre la Ética y la Responsabilidad Social Empresarial. Universidad La Salle, 1-50.

Marrero, M. (2013). La importancia de la información para la toma de decisiones. REMIJ, 14(2), 1-3.

Martín, P., \& Aroca, B. (2016). Corporate social responsibility in family SMEs: A comparative study. European Journal of Family Business, 6(1), 21-31. https://doi.org/10.1016/j.ejfb.2016.05.002 
Muñoz, B. (2018). Ventajas y desventajas del muestreo probabilístico y no probabilístico en investigaciones científicas. Universidad Técnica de Machala.

Ochoa, E. (2017). Incidencia de las Prácticas de Responsabilidad Social Empresarial en la Universidad Militar Nueva Granada.

Pasquel, G., Báez, V., Pauker, L., \& Apolo, D. (2016). Percepción, activos intangibles y stakeholders: modelo para el análisis de imagen corporativa. Revista Mediterránea de Comunicación, 7(1), 179-196.

Quero, M. (2010). Confiabilidad y coeficiente Alpha de Cronbah. TELOS, 3, 1547-1550. https://doi.org/10.1109/igarss.2004.1370608

Sampieri, H., Fernández, C., \& Baptista, P. (2014). Metodología de la investigación (M. Hill (ed.); sexta edic).

Schwalb, M. (2011). RSE en América Latina. La responsabilidad social de la empresa en América Latina (pp. 87-110).

SEPS. (2015). Código orgánico monetario y financiero. Superintendencia de Economía Popular y Solidaria.

SEPS. (2017). Cooperativas hacia la responsabilidad social - SEPS. Superintendencia de Economía Popular y Solidaria.

SEPS. (2021). Aprende un poco más sobre el sector: ¿Qué es la economía popular y solidaria? - SEPS. Superintendencia de Economía Popular y Solidaria.

Server, R., \& Villalonga, I. (2015). La responsabilidad social corporativa y su gestión integrada. CIRIEC-España, Revista de Economía Pública, Social y Cooperativa, 53, 137-161.

Teng, J., Wei, M., Yuen, S., \& Suan, W. (2000). Enhancing corporate image in organisations. Management Research News, 23(5-6), 2854. https://doi.org/10.1108/01409170010782037

Vélez, X., \& Cano, E. (2016). Los diferentes tipos de responsabilidad social y sus implicaciones éticas. Dominio de Las Ciencias, 2(3), 117-126.

Virvilaite, R., \& Daubaraite, U. (2011). Corporate Social Responsibility in Forming Corporate Image. Engineering Economics, 22(5), 534-543. https://doi.org/10.5755/j01.ee.22.5.972 
Zhivkova, S. (2019). The Companies Behavior in the Context of Sustainable Development. Economic Alternatives, 2, 275-286. 